\title{
Controle químico da Grapholita molesta (Busck) (Lepidoptera: Tortricidae) na cultura do pessegueiro
}

\author{
Chemical control of Grapholita molesta (Busck) (Lepidoptera: Tortricidae) \\ in peach orchards
}

\section{Cristiano João Arioli ${ }^{1}$ Marcos Botton ${ }^{2}$ Geraldo Andrade Carvalho ${ }^{3}$}

\section{RESUMO}

A mariposa oriental Grapholita molesta (Busck) (Lepidoptera: Tortricidae) é a principal praga da cultura do pessegueiro no Brasil. O controle do inseto depende basicamente do emprego de inseticidas de alta toxicidade e baixa seletividade aos inimigos naturais. Este trabalho avaliou os inseticidas etofemprox (Trebon $100 \mathrm{SC}, 100$ e $150 \mathrm{~mL} 100 \mathrm{~L}^{-1}$ ), benzoato de emamectina (Proclaim $5 \mathrm{SG}, 10$ e $15 \mathrm{~g} 100 \mathrm{~L}^{-1}$ ) + óleo mineral (Assist, 250mL 100L $\mathrm{L}^{-1}$ ), metoxyfenozide (Intrepid $240 \mathrm{SC}, 40 \mathrm{e}$

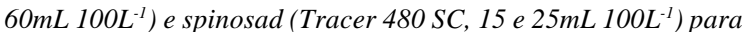
o controle da G. molesta na cultura do pessegueiro. No experimento de laboratório, somente o etofemprox apresentou baixa mortalidade $( \pm 50 \%)$ de lagartas. Em pomar comercial, todos os inseticidas e doses testadas reduziram o nível de injúria nos ponteiros em nível superior a $80 \%$. Os inseticidas avaliados apresentam características desejáveis para uso no manejo integrado da G. molesta, destacando-se a baixa toxicidade e reduzida dose de aplicação, o que minimiza os riscos ao homem $e$ ambiente.

Palavas-chave: mariposa oriental; controle químico; produção integrada de frutas.

\section{ABSTRACT}

Oriental fruit moth Grapholita molesta (Busck) (Lepidoptera: Tortricidae) is the most important peach pest in Brazil. Pest management is based on chemical control using insecticides with high toxicity and low selectivity to natural enemies. Etofemprox (Trebon $100 \mathrm{SC}, 100$ and $150 \mathrm{~mL} 100 \mathrm{~L}^{-1}$ ), emamectin benzoato (Proclaim $5 \mathrm{SG}, 10$ and $15 \mathrm{~g} 100 \mathrm{~L}^{-1}$ ) associated to mineral oil (Assist, 250mL 100L $\mathrm{L}^{-1}$ ), metoxyfenozide (Intrepid $240 \mathrm{SC} 40$ and 60mL 100L $\mathrm{L}^{-1}$ ), spinosad (Tracer $480 \mathrm{SC}$ 15 and $25 \mathrm{~mL} 100 \mathrm{~L}^{-1}$ ) and fosmet (Imidan $500 \mathrm{PM}, 200 \mathrm{~g} 100 \mathrm{~L}^{-1}$ ) were evaluated in laboratory and field conditions to G. molesta control. In laboratory, only etofemprox resulted in low mortality $( \pm 50 \%)$ in residual bioassay. In commercial peach orchards, all insecticides reduced pest damage (> 80\%). All insecticides shows characteristics for use in the integrated management of G. molesta including low toxicity and reduced application dose.

Key words: oriental fruit moth; chemical control; integrated fruit production.

\section{INTRODUÇÃO}

A Grapholita molesta (Busck) (Lepidoptera: Tortricidae), conhecida comumente por mariposa oriental, é nativa do continente asiático (SALLES, 1998). Foi introduzida no Brasil em 1929, no Estado do Rio Grande do Sul (SILVA et al., 1962) de onde se dispersou para os demais estados das regiões centro-sul, sendo considerada atualmente uma das principais pragas do pessegueiro e de outras frutiferas de clima temperado (HICKEL \& DUCROQUET, 1998; RIBEIRO, 1999; SOUZA et al., 2000; BOTTON et al., 2001; NORA \& SUGIURA, 2001; KOVALESKI \& RIBEIRO, 2003).

O controle químico, através do emprego de inseticidas fosforados e piretróides, tem sido o método mais utilizado pelos produtores para o manejo da praga (SANTA CECÍLIA \& DE SOUZA, 1985; SALLES, 1998). No entanto, existe uma preocupação crescente entre técnicos e produtores para racionalizar e/ou substituir o emprego destes insumos,

\footnotetext{
${ }^{1}$ Engenheiro Agrônomo, Doutorando em Fitossanidade, Universidade Federal de Pelotas (UFPel), CP 354, 96010-900, Pelotas, RS. ${ }^{2}$ Engenheiro Agrônomo, Pesquisador Doutor Entomologia, Embrapa Uva e Vinho, CP 130, 95.700-000, Bento Gonçalves, RS. E-mail: marcos@cnpuv.embrapa.br.Autor para correspondência

${ }^{3}$ Engenheiro Agrônomo, Professor Doutor Entomologia, Universidade Federal de Lavras (UFLA), CP 37, 37200-000, Lavras, MG.
} 
especialmente nos agroecossistemas frutícolas conduzidos sob sistemas de produção integrada (TITI et al., 1995). Dentre os motivos que promovem a necessidade urgente de substituição desses insumos, destacam-se os efeitos secundários sobre a entomofauna benéfica, riscos de intoxicação dos aplicadores e contaminação ambiental (GONRING et al., 1999; BOTTON et al., 2001). Além disso, são cada vez maiores as restrições ao uso de inseticidas fosforados devido à elevada toxicidade e carência, principalmente quando são empregados no período de pré-colheita das frutas (NORMAS, 2001).

Dentre as novas alternativas para o controle químico da mariposa oriental, destacam-se os lagarticidas específicos como metoxyfenozide e o benzoato de emamectina (VITTONE et al., 1996; GRÜTZMACHER et al., 1999; MARZOCCHI, 1999; VISIGALLI et al, 2000). Como vantagens desses produtos, ressalta-se a dose reduzida, baixa toxicidade e carência, além da especificidade sobre lagartas, reduzindo os efeitos tóxicos sobre organismos nãoalvo (MORANDO et al., 1990; LEIBBE et al., 1995; CARLSON et al., 2001). Esses compostos representam uma alternativa para o controle das primeiras gerações da mariposa oriental na cultura, especialmente quando o inseto é o único causador de danos.

Além desses inseticidas, é fundamental que sejam avaliadas novas alternativas para substituir os inseticidas piretróides e o dimetoato no período de pré-colheita das frutas (NORMAS, 2001). Dentre os novos inseticidas que possuem potencial para serem empregados nessa fase, destacam-se o spinosad, produto derivado da fermentação do actinomiceto Sacharopolyspora spinosa (THOMPSON \& HUTCHINS, 1999) e o etofemprox, composto à base de carbono, oxigênio e hidrogênio (YOSHIMOTO et al., 1989).

Este trabalho teve por objetivo avaliar novos inseticidas que possam ser empregados no controle da mariposa oriental na cultura do pessegueiro, visando substituir os fosforados atualmente empregados no controle da praga.

\section{MATERIAL E MÉTODOS}

Os experimentos foram conduzidos no Laboratório de Entomologia da Embrapa Uva e Vinho (temperatura de $25 \pm 2^{\circ} \mathrm{C}$, umidade relativa de $70 \pm 10 \%$ e fotofase de 16 horas) e em pomar comercial de pessegueiro localizado no Distrito de Pinto Bandeira, pertencente ao município de Bento Gonçalves, RS (latitude $29^{\circ} 07^{\prime}$ Sul, longitude $51^{\circ} 26^{\prime}$ 'Oeste e altitude aproximada de 725 metros). Os tratamentos constaram de diferentes inseticidas e concentrações (Tabela 1 e 2), mantendo-se um tratamento testemunha onde foi aplicado somente água.

\section{Bioensaio em laboratório}

Lagartas de $\boldsymbol{G}$. molesta foram coletadas em ponteiros na cultura do pessegueiro, em pomar sem aplicação de inseticidas, em Bento Gonçalves, RS, sendo colocadas no interior de caixa térmica para transporte até o laboratório, onde, quatro horas após, foram retiradas e separadas por tamanho (entre 4 e 8 $\mathrm{mm}$ ), equivalendo ao 3o - 4o ínstar. Pêssegos da cultivar Premier, colhidos em ponto de maturação no mesmo pomar, em outubro de 2002, foram levados ao laboratório e mergulhados por 5 segundos, em um litro de solução do respectivo tratamento contido em recipiente de vidro. Após tratados, os frutos foram deixados à sombra por duas horas para secagem e logo transferidos a recipientes do tipo copo plástico (capacidade de $300 \mathrm{~mL}$ ) medindo aproximadamente $10,0 \mathrm{~cm}$ de altura $\mathrm{x} 5,0 \mathrm{~cm}$ de diâmetro. Cada fruto foi infestado com uma lagarta e o recipiente foi tampado com tecido tipo voil.

O delineamento experimental foi o inteiramente casualizado, estabelecendo-se dez repetições por tratamento, sendo cada parcela constituída por três recipientes contendo um fruto e uma lagarta cada. A avaliação da mortalidade foi realizada 48 e 96 horas após a infestação, sendo considerados mortos aqueles insetos que não apresentaram reação ao toque de um pincel. Os dados do número de lagartas sobreviventes foram transformados para $\sqrt{y+0,5}$, comparando-se as médias pelo teste de SCOTT-KNOTT (1974) a um nível de $5 \%$ de probabilidade de erro. A porcentagem de controle foi calculada pela fórmula de ABBOTT (1925).

Experimento em pomar comercial

O experimento foi conduzido em pomar de pessegueiro com um ano de idade da cultivar Chimarrita, com infestação natural da praga, com plantas de $1,5 \mathrm{~m}$ de altura, plantadas no espaçamento $3,0 \mathrm{~m} \times 4,0 \mathrm{~m}$. Os inseticidas foram aplicados em intervalos de dez dias, sendo a primeira aplicação realizada em 13 de dezembro de 2002 e a última em 3 de janeiro de 2003, totalizando três pulverizações. Antes da primeira aplicação, foram retirados todos os ponteiros danificados pela praga nas planas. As aplicações foram realizadas com um pulverizador costal manual, modelo PJH 20, equipado com bico de cone vazio, modelo JA-1,5, da empresa Máquinas Agrícolas Jacto S.A, Pompéia, SP. As plantas foram 
pulverizadas até o ponto de escorrimento, gastandose em média $75 \mathrm{~mL}$ de calda planta ${ }^{-1}$, eqüivalendo a $60 \mathrm{~L} \mathrm{ha}^{-1}$ (plantas jovens).

O delineamento experimental foi o de blocos inteiramente casualizados com quatro repetições. Cada parcela foi formada por quatro plantas. A avaliação do efeito dos inseticidas foi realizada dez dias após a última aplicação (13 de janeiro), contando-se o número de ponteiros danificados pela praga nas duas plantas centrais de cada parcela. A porcentagem de ponteiros danificada foi transformada para arc seno $\sqrt{\% / 100}$, a comparação de médias foi realizada pelo teste de SCOTT-KNOTT (1974), em um nível de 5\% de probabilidade de erro e a porcentagem de controle foi calculada pela fórmula de ABBOTT (1925).

\section{RESULTADOS E DISCUSSÃO}

Bioensaio em laboratório

Quarenta e oito horas após a aplicação dos inseticidas, o spinosad e o benzoato de emamectina provocaram mortalidade superior a $64 \%$ (Tabela 1), valor próximo ao observado com o tratamento fosmet, inseticida mais utilizado no controle da G. molesta na região. Nesta avaliação, os inseticidas metoxyfenozide e etofemprox demonstraram baixa eficiência de controle, entre 17,86 e 35,71\%.

$\mathrm{Na}$ avaliação realizada 96 horas após a aplicação (HAA), com exceção do etofemprox, os inseticidas controlaram o inseto em níveis superiores a $80 \%$ (Tabela 1), sendo considerados eficientes no controle da mariposa oriental. A diferença na mortalidade das lagartas entre 48 e 96 HAA, observada com o metoxyfenozide (regulador de crescimento), deve-se ao modo de ação desse inseticida, que é mais lento que os produtos neurotóxicos (DHADIALLA et al., 1998). TRISYONO \& CHIPPENDALE (1997), observando o efeito do metoxyfenozide sobre lagartas de Ostrinia nubilalis (Hubner) (Lepidoptera: Pyralidae), verificaram baixa mortalidade do inseto em laboratório, 48 horas após aplicação. Porém, passadas 104 horas, esta chegou a 70\%. Constataram ainda que o inseticida paralisou a alimentação das lagartas 8 horas após a aplicação, induzindo a uma muda prematura e letal. SUN \& BARRETT (1999) e SUN et al. (2000), estudando a ação dos produtos metoxyfenozide e tebufenozide sobre Cydia pomonella (Linnaeus), Argyrotaenia velutinana (Walker) e Choristoneura rosaceana (Harris) (Lepidoptera: Tortricidae), pragas que atacam a cultura da macieira, observaram efeito sub-letal, caracterizado pela redução na fecundidade e fertilidade dos ovos.
WANNER et al. (2000) avaliaram a eficiência de spinosad no controle de lagartas de Lymantria dispar (Linnaeus) (Lepidoptera: Lymantriidae) e verificaram que o produto foi ativo sobre lagartas de $2^{\circ}$ ínstar. $\mathrm{O}$ inseticida paralisou a atividade das lagartas 8 horas após o tratamento, provocando elevada mortalidade nove dias do início do teste. Os autores também observaram ação de contato, embora, segundo SPARKS et al. (1998), a maior atividade do produto seja obtida quando o composto é ingerido. HILL \& FOSTER (2000) constataram que o spinosad foi eficiente sobre lagartas de Plutella xylostella (Linnaeus) (Lepidoptera: Plutellidae), obtendo $100 \%$ de mortalidade 48 horas após a aplicação. Observaram também que, 72 horas após o tratamento dos adultos, o spinosad promoveu $100 \%$ de mortalidade, demonstrando alta eficiência sobre os diferentes estádios de desenvolvimento da praga.

\section{Experimento em pomar comercial}

$\mathrm{O}$ ataque da mariposa oriental aos ponteiros foi significativamente reduzido em todas as parcelas tratadas com os inseticidas (Tabela 2). Os produtos etofemprox $\left(150 \mathrm{~mL} \quad 100 \mathrm{~L}^{-1}\right) \quad \mathrm{e}$

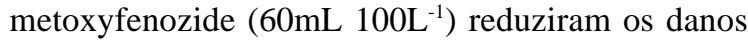
aos ponteiros acima de $90 \%$, demonstrando elevada eficiência em condições de campo. Além desses, benzoato de emamectina, metoxyfenozide $(40 \mathrm{~mL}$ $\left.100 \mathrm{~L}^{-1}\right)$ e spinosad $\left(15\right.$ e $\left.25 \mathrm{~mL} 100 \mathrm{~L}^{-1}\right)$ também proporcionaram redução no ataque acima de $80 \%$, semelhante à demonstrada pelo fosmet. $\mathrm{O}$ etofemprox na menor dose reduziu o dano da praga em aproximadamente $70 \%$.

De maneira similar ao observado em laboratório, o benzoato de emamectina, spinosad e metoxifenozide controlaram com eficiência a mariposa oriental, apresentando potencial para substituir os fosforados e piretróides no controle dessa praga na cultura do pessegueiro. O etofemprox, diferente do observado em laboratório, demonstrou ser eficiente em condições de campo (Tabela 2). Em hipótese, as pulverizações realizadas em pomar comercial são mais eficazes que no laboratório, pois atingem diretamente todas as fases do inseto, principalmente lagartas de $1^{\circ}$ e $2^{\underline{o}}$ ínstares, possibilitando, também, efeito repelente sobre adultos, fazendo com que as fêmeas não ovipositem sobre as plantas tratadas.

Os resultados deste trabalho assemelhamse aos obtidos por GRÜTZMACHER et al. (1999) e VISIGALLI et al. (2000), que verificaram que o metoxyfenozide foi eficiente na redução dos danos causados pela $\boldsymbol{G}$. molesta em ponteiros e frutos nas 
Tabela 1 - Número (N) $( \pm \mathrm{EP})$ de lagartas vivas do terceiro ao quarto instar de Grapholita molesta por tratamento e eficiência de controle $(\% \mathrm{C})$ após contato com pêssegos tratados. Bento Gonçalves, 2003.

\begin{tabular}{|c|c|c|c|c|c|c|c|c|}
\hline \multirow{2}{*}{$\begin{array}{l}\text { Produto } \\
\text { Comercial }\end{array}$} & \multirow{2}{*}{ Ingrediente } & \multicolumn{2}{|c|}{ Dose $^{1}$} & \multicolumn{3}{|c|}{$48 \mathrm{HAA}^{2}$} & \multicolumn{2}{|c|}{$96 \mathrm{HAA}^{2}$} \\
\hline & & ia & $\mathrm{pc}$ & $\mathrm{N}$ & & $\% \mathrm{C}^{5}$ & $\mathrm{~N}$ & $\% \mathrm{C}^{5}$ \\
\hline Imidan $500 \mathrm{PM}$ & Fosmet & 100,0 & 200,0 & $0,8 \pm 0,20$ & $d A^{4}$ & 71,43 & $0,5 \pm 0,22 \mathrm{cA}^{4}$ & 80,77 \\
\hline Intrepid $240 \mathrm{SC}$ & Metoxi fenozide & 9,6 & 40,0 & $2,3 \pm 0,26$ & $\mathrm{bA}$ & 17,86 & $0,3 \pm 0,21 \quad \mathrm{cB}$ & 88,46 \\
\hline Intrepid $240 \mathrm{SC}$ & Metoxi fenozide & 14,4 & 60,0 & $2,3 \pm 0,21$ & bA & 17,86 & $0,3 \pm 0,21 \quad \mathrm{cB}$ & 88,46 \\
\hline Proclain $5 \mathrm{SG}^{3}$ & Benzoato de emamectina & 0,50 & 10,0 & $1,0 \pm 0,30$ & $\mathrm{dA}$ & 64,29 & $0,2 \pm 0,13 \quad \mathrm{cB}$ & 92,31 \\
\hline Proclain 5 SG & Benzoato de emamectina & 0,75 & 15,0 & $0,9 \pm 0,31$ & dA & 67,86 & $0,2 \pm 0,13 \quad \mathrm{cB}$ & 92,31 \\
\hline Tracer $480 \mathrm{SC}$ & Spinosad & 7,20 & 15,0 & $0,8 \pm 0,25$ & dA & 71,43 & $0,5 \pm 0,22 \quad \mathrm{cA}$ & 80,77 \\
\hline Tracer $480 \mathrm{SC}$ & Spinosad & 12,00 & 25,0 & $0,4 \pm 0,22$ & dA & 85,71 & $0,2 \pm 0,13 \mathrm{cA}$ & 92,31 \\
\hline Trebon $100 \mathrm{SC}$ & Etofemprox & 10,0 & 100,0 & $1,8 \pm 0,29$ & $\mathrm{cA}$ & 35,71 & $1,3 \pm 0,26 \quad \mathrm{bA}$ & 50,00 \\
\hline Trebon $100 \mathrm{SC}$ & Etofemprox & 15,0 & 150,0 & $1,8 \pm 0,25$ & $\mathrm{cA}$ & 35,71 & $1,2 \pm 0,33 \quad b B$ & 53,85 \\
\hline Testemunha & Água & - & - & $2,8 \pm 0,13$ & aA & - & $2,6 \pm 0,22$ aA & - \\
\hline
\end{tabular}

${ }^{1}$ Gramas ou $\mathrm{mL}$ de ingrediente ativo (ia) ou produto comercial (pc) por 100 litros de água.

${ }^{2}$ Horas após a aplicação dos inseticidas.

${ }^{3}$ Associado ao óleo mineral (Assist 0,25\%).

${ }^{4}$ Médias não seguidas por mesmas letras maiúsculas na linha e minúsculas nas colunas diferem entre si pelo teste de Scott-Knott em nível de $5 \%$ de probabilidade de erro.

${ }^{5}$ Eficiência de controle de acordo com fórmula de Abbott (1925).

Tabela 2 - Porcentagem de ponteiros danificados (\%PD) ( $₫ \mathrm{EP})$ por Grapholita molesta após três aplicações de inseticidas, em pomar comercial de pessegueiro da cultivar Chimarrita. Bento Gonçalves, 2003.

\begin{tabular}{|c|c|c|c|c|c|c|}
\hline \multirow{2}{*}{$\begin{array}{l}\text { Produto } \\
\text { Comercial }\end{array}$} & \multirow{2}{*}{ Ingrediente ativo } & \multicolumn{2}{|c|}{$\operatorname{Dose}^{1}$} & \multirow{2}{*}{$\% \mathrm{PD}$} & & \multirow{2}{*}{$\%$ PRD3 } \\
\hline & & ia & $\mathrm{pc}$ & & & \\
\hline Imidan $500 \mathrm{PM}$ & Fosmet & 100 & 200 & $3,9 \pm 1,35$ & $b^{2}$ & 80,0 \\
\hline Intrepid $240 \mathrm{SC}$ & Metoxi fenozide & 9,6 & 40 & $2,9 \pm 0,73$ & $\mathrm{~b}$ & 85,3 \\
\hline Intrepid $240 \mathrm{SC}$ & Metoxi fenozide & 14,4 & 60 & $1,4 \pm 1,05$ & $\mathrm{~b}$ & 92,8 \\
\hline Proclain $5 \mathrm{SG}^{4}$ & Benzoato de emamectina & 0,50 & 10 & $3,6 \pm 1,37$ & $\mathrm{~b}$ & 81,9 \\
\hline Proclain $5 \mathrm{SG}^{4}$ & Benzoato de emamectina & 0,75 & 15 & $2,8 \pm 1,67$ & $\mathrm{~b}$ & 85,7 \\
\hline Tracer $480 \mathrm{SC}$ & Spinosad & 7,20 & 15 & $3,4 \pm 0,8$ & $\mathrm{~b}$ & 82,6 \\
\hline Tracer $480 \mathrm{SC}$ & Spinosad & 12,00 & 25 & $3,1 \pm 1,41$ & $\mathrm{~b}$ & 84,3 \\
\hline Trebon $100 \mathrm{SC}$ & Etofemprox & 10,0 & 100 & $6,2 \pm 2,58$ & $\mathrm{~b}$ & 68,2 \\
\hline Trebon $100 \mathrm{SC}$ & Etofemprox & 15,0 & 150 & $1,3 \pm 1,33$ & $\mathrm{~b}$ & 93,2 \\
\hline Testemunha & água & - & - & $19,6 \pm 5,12$ & $\mathrm{a}$ & - \\
\hline
\end{tabular}

${ }^{1}$ Gramas ou mL de ingrediente ativo (ia) ou produto comercial (pc) por 100 litros de água.

${ }^{2}$ Médias não seguidas da mesma letra diferem entre si pelo teste de Scott-Knott em nível de 5\% de probabilidade de erro.

${ }^{3}$ Eficiência de controle de acordo com fórmula de Abbott (1925).

${ }^{4}$ Associado ao óleo mineral (Assist 0,25\%).

culturas da pereira e pessegueiro, respectivamente. Resultados semelhantes foram obtidos por CIVOLANI et al. (1999; 2001a; b), BOSELLI \& SCANNAVINI (2001) e BOSELLI \& VERGNANI (2001) que verificaram que o metoxyfenozide também provocou elevada mortalidade de lagartas de Pandemis cerasana (Hübner), C. pomonella e Lobesia botrana (Denis e Schiffermüller) (Lepidoptera: Tortricidae).
GRAVENA et al. (2002) avaliaram, em pomar comercial, o efeito do spinosad sobre a lagartaminadora-dos-citros Phyllocnistis citrella (Stainton) (Lepidoptera: Gracilariidae) e verificaram uma eficiência no controle entre 91 e $95 \%$. WANNER et al. (2000) observaram que o spinosad aplicado sobre plantas de vidoeiro (bétula) promoveu $100 \%$ de mortalidade de $\boldsymbol{L}$. dispar três dias após o tratamento. Sobre Spodoptera littoralis (Boisduval) (Lepidoptera:

Ciência Rural, v.34, n.6, nov-dez, 2004. 
Noctuidae), na Itália, esse produto reduziu significativamente os danos ocasionados por essa lagarta na cultura da alface (SANNINO, 2001).

Em relação ao etofemprox, MORANDO et al. (1990) e TOSI et al. (1999) concluíram que o inseticida controlou eficientemente Eupoecilia ambiguella (Hübner) (Lepidoptera: Tortricidae) e $\boldsymbol{L}$. botrana na cultura da videira. MORANDO et al. (1990) demonstraram que o produto reduziu significativamente o número de lagartas de $\boldsymbol{E}$. ambiguella por cacho de uva (96\%) e o dano ocasionado pelas mesmas $(90 \%)$ em comparação à testemunha. Além do bom controle das pragas, os autores relataram que o produto não alterou o equilíbrio biológico do vinhedo, já que, na contagem de ácaros fitófagos, os valores não diferiram significativamente daquele obtido na área testemunha.

A respeito do benzoato de emamectina, LEIBBE et al. (1995) observaram que o inseticida, aplicado em campos de produção de repolho, na região da Flórida, Estados Unidos, foi eficiente no controle de $\boldsymbol{P}$. xylostella. Os autores verificaram que o composto promoveu baixos níveis de infestação da praga, havendo maior porcentagem de cabeças de repolho com características comerciais. JANSSON et al. (1997), em dois estudos de campo, constataram que o produto foi efetivo no controle de $\boldsymbol{H}$. zea e $S$. exigua, demonstrando ter potencial para o controle desses lepidópteros.

Na Região da Serra Gaúcha, são observados quatro picos populacionais durante o ciclo de produção do pessegueiro (BOTTON et al., 2001). Considerando o período de ocorrência da mariposa oriental na cultura e o modo de ação dos inseticidas avaliados, metoxyfenozide e benzoato de emamectina, por serem lagarticidas específicos, devem ser direcionados ao controle das primeiras gerações de lagartas ocorrentes no pomar, quando somente a mariposa oriental é encontrada, causando danos à cultura. Devido ao modo de ação (basicamente por ingestão), a aplicação deve ser realizada logo após a eclosão das lagartas, antes de sua entrada nos ponteiros (brotos). Assim, é fundamental a existência de um bom sistema de monitoramento para se obter um controle eficiente da praga (BOTTON et al., 2001). Já em relação aos produtos spinosad e etofemprox, pelo maior espectro de ação, podem ser direcionados para a fase de pré-colheita, visto a possibilidade de controle conjuto dos adultos da mosca-das-frutas sul-americana Anastrepha fraterculus (Wiedemann, 1830) (Diptera: Tephritidae) que também ocorre nesta fase (SCOZ, 2003). Os inseticidas benzoato de emamectina, etofemprox, metoxyfenozide e spinosad apresentam- se como novas alternativas para o controle da mariposa oriental na cultura do pessegueiro, apresentando menor toxicidade e reduzida dose de aplicação quando comparados aos fosforados.

\section{CONCLUSÃO}

Os inseticidas benzoato de emamectina (Proclaim 5 SG, 10 e 15g 100L-1) associado ao óleo mineral (Assist, 250mL 100L $\mathrm{L}^{-1}$ ), etofemprox (Trebon

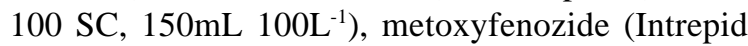

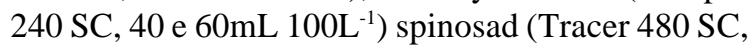

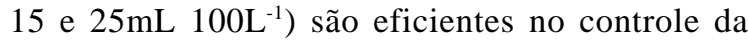
Grapholita. molesta na cultura do pessegueiro.

\section{REFERÊNCIAS BIBLIOGRÁFICAS}

ABBOTT, W.S. A method of computing the effectiveness of an insecticide. Journal of Economic Entomology, Lanham, v.18, n.1, p.265-267, 1925.

BOSELLI, M.; SCANNAVINI, M. Lotta alla tignoletta della vite in Emilia-Romana. L'Informatore Agrario, Verona, n.19, p.97-100, 2001.

BOSELLI, M.; VERGNANI, S. Attività di alcuni insetticidi nei confronti della prima generazione di Cydia pomonella L. Informatore Fitopatologico, Bologna, n.6, p.40-46, 2001

BOTTON, M.; ARIOLI, C.J.; COLLETTA, V.D. Monitoramento da mariposa oriental Grapholita molesta (Busck, 1916) na cultura do pessegueiro. Bento Gonçalves : Embrapa-CNPUV, 2001. 4p. (Embrapa-CNPUV. Comunicado Técnico, 38).

CARLSON, G.R. et al. The chemical and biological properties of metoxifenozide, a new insecticidal ecdysteroid agonist. Pest Management Science, Hoboken, v.57, p.115-119, 2001.

CIVOLANI, S. et al. Tebufenozide e metoxifenozide (MAC) momento di applicazione su Pandemis cerasana. Informatore Fitopatologico, Bologna, n.9, p.55-62, 1999.

CIVOLANI, S.; VERGANI, S.; PASQUALINI, E. Tecnica di difesa per la gernerazione svernate de Pandemis cerasana Hüber (Lepidoptera: Tortricidae) su melo in Emilia-Romagna. Informatore Fitopatologico, Bologna, n.10, p.44-48, 2001a.

CIVOLANI, S.; VERGANI, S.; PASQUALINI, E. Efficacia del metoxifenozide su Cydia pomonella. L'Informatore Agrario, Verona, n.34, p.63-64, 2001b.

DHADIALLA, T.S.; CARLSON, G.R.; LE, D. New insecticides with ecdysteroidal and juvenile hormone activity. Annual Review of Entomology, Palo Alto, v.43, p.545-569, 1998.

GONRING, A.H.R. et al. Seletividade de inseticidas, utilizados no controle de Grapholita molesta (Busck) (Lepidoptera: Olethreutidae) em pêssego, a Vespidae predadores. Anais da Sociedade Entomológica do Brasil, Londrina, v.28, n.2, p.301306, 1999.

GRAVENA, S. et al. Efeito de dosess de spinosade adicionado de óleo mineral no controle da lagarta-minadora-dos-citros. 
Revista Laranja, Cordeirópolis, v.23, n.1, p.155-165, 2002.

GRÜTZMACHER, et al. Eficiência dos inseticidas fisiológicos Mimic 240 SC (tebufenozide) e Intrepid 240 SC (metoxifenozide) no controle da mariposa oriental Grapholita molesta (Busck, 1916) (Lepidoptera: Tortricidae) na cultura da pereira. Revista Brasileira de Agrociência, Pelotas, v.5, n.3, p.211-215, 1999.

HICKEL, E.R.; DUCROQUET, J.H.J. Monitoramento e controle da grafolita ou mariposa oriental no Alto Vale do Rio do Peixe. Agropecuária Catarinense, Florianópolis, v.11, n.2, p.8-11, 1998.

HILL, T.A.; FOSTER, R.E. Effect of insecticides on the diamondback moth (Lepidoptera: Plutellidae) and its parasitoid Diadegma insulare (Hymenoptera: Ichneumonidae). Journal of Economic Entomology, Lanham, v.93, n.3, p.763-768, 2000.

JANSSON, R.K. et al. Efficacy of solid formulation of emamectin benzoate controlling lepidopterous pest. Florida Entomology, Lutz, v.79, n.1, p.434-449, 1997.

KOVALESKI, A; RIBEIRO, L.G. Manejo de pragas na produção integrada de maçã. In: PROTAS, J.F. da S.; SANHUEZA, R.M.V. Produção integrada de frutas: o caso da maçã no Brasil. Bento Gonçalves : EMBRAPA UVA E VINHO, 2003. p.61-68.

LEIBBE, G.L. et al. Efficacy of emamectin benzoate and Bacillus thuringiensis at controlling diamondback moth (Lepidoptera: Plutellidae) populations on cabbage in Florida. Florida Entomologist, Lutz, v.78, n.1, p.82-96, 1995.

MARZOCCHI, L. Lotta integrata contro cidia e anarsia. Terra e Vita, Bologna, n.11, p.38-40, 1999.

MORANDO, A.; BEVIONE, D.; MORINO, G. Prove di controlle delle tignole della vite côn prodotti tradizionali e regolatori di crescita. L'Informatore Agrario, Verona, n.16, p.141-145, 1990.

NORA, I.; SUGIURA, T. Pragas da pereira. In: EPAGRI (Ed.). Nashi, a pêra japonesa. Florianópolis : EPAGRI/JICA, 2001. p.261-321.

NORMAS de produção integrada de pêssego (PIP): versão II. Pelotas : UFPel/Embrapa/UFRGS/URCAMP, 2001. 52p.

RIBEIRO, L.G. Principais pragas da macieira. In: BONETTI, J.I.S. da.; RIBEIRO, L.G; KATSURAYAMA, V. Manual de identificação de doenças e pragas da macieira. Florianópolis : EPAGRI, 1999. Cap.3, p.97-149.

SALLES, L.A.B. de. Principais pragas e seu controle. In: MEDEIROS, C.A.B.; RASEIRA, M. do C. A cultura do pessegueiro. Brasília : EMBRAPA- CPACT, 1998. Cap.8, p.206242.

SANNINO, L. Efficacia contro Spodoptera littoralis di un nuovo formulato. L'Informatore Agrario, Verona, n.5, p.74-76, 2001.

SANTA CECÍLIA, L.V.C.; DE SOUZA, J.C. de. Pragas das frutiferas de clima temperado. Informe Agropecuário, Belo Horizonte, v.11, n.125, p.43-56, 1985.
SCOTT, A.J.; KNOTT, M.A. A cluster analyses method for grouping means in the analyses of variance. Biometrics, v.30, p.502-512, 1974.

SCOZ, P.L. Avaliação de atrativos alimentares, armadilhas e inseticidas para o monitoramento e controle de Anastrepha fraterculus (Wiedemann, 1830) (Diptera: Tephritidae). 2003. 52f. Dissertação (Mestrado em Fitossanidade) - Curso de Pósgraduação em Fitossanidade, Universidade de Pelotas.

SILVA, A.G. et al. Quarto catálogo dos insetos que vivem nas plantas do Brasil. Rio de Janeiro : [s.n.], 1962. 622p.

SOUZA, B.; SANTA-CECÍLIA, L.V.C.; DE SOUZA, L.O.V. Ocorrência de danos de Grapholita molesta (Busck) (Lepidoptera: Tortricidae) em pessegueiros no município de Caldas - MG. Anais da Sociedade Entomológica do Brasil, Jaboticabal, v.29, n.1, p.185-188, 2000.

SPARKS, T.C. et al. Biological activity of the spinosys, new fermentation derived insect control agents, on tobacco budworm (Lepidoptera: Noctuidae) larvae. Journal of Economic Entomology, Lanham, v.91, p.1277-1283, 1998.

SUN, X.; BARRETT, B.A Fecundidad and fertility changes in adult codling moth (Lepidoptera: Tortricidae) exposed to surfaces treated with tebufenozide and metoxifenozide. Journal of Economic Entomology, Lanham, v.92, n.5, p.1039-1044, 1999.

SUN, X.; BARRETT, B.A; BIDDINGER, D.J. Fecundidad and fertility changes in adult leafrollers exposed to surfaces treated with ecdysteroid agonists tebufenozide and metoxifenozide. Entomologia Experimentalis et Applicata, v.94, p.75-83, 2000.

THOMPSON, G.; HUTCHINS, S. Spinosade. Pesticide Outlook, Saskatoon, v.10, n.2, p.78-81, 1999.

TITI, A. EL.; BOLLER, E.F.; GENDRIER, J.P. (Ed.). Producción integrada: principios y directrices técnicas. [S.1.]: IOBC/WPRS, 1995. 22p. (Bulletin, 18).

TOSI, L. et al. Efficacia di alcuni insetticidi sulla tignoletta della vite. L'Informatore Agrario, Verona, n.26, p.59-61, 1999.

TRISYONO, A.; CHIPPENDALE, M. Effect of the nonsteroidal ecdysone agonists, metoxifenozide and tebufenozide, on the European corn borer (Lepidoptera: Pyralidae). Ecotoxicology, v.90, n.6, p.1486-1492, 1997.

VISIGALLI, T. et al. Eficacia di alcuni insetticidi contro la tignola orientale del pesco. L'Informatore Agrario, Verona, n.21, p.85$88,2000$.

VITTONE, G.; ASTESANO, B.; AIMAR, S. L'impiego di prodotti chitino-inibitori contro la cidia del pesco. L'Informatore Agrario, Verona, n.19, p.62-64, 1996.

WANNER, K.W.; HELSON, B.V.; HARRIS, B.J. Laboratory and field evaluation of spinosad against the gypsy moth, Lymantria dispar. Pest Management Science, Hoboken, v.56, p.855-860, 2000.

YOSHIMOTO, T. et al. Development of new inseticide, Etofenprox. Journal of Pest Science, v.14, n.2, p.259-268, 1989. 